\title{
CP parameters of the $B$ systems from Tevatron
}

\author{
S. Burdin ${ }^{\mathrm{a}}$ on behalf of CDF and DØ collaborations

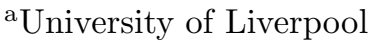 \\ Recent results on CP parameters of the $B$ systems obtained by the CDF and D $\varnothing$ collaborations using the data \\ samples collected at the Tevatron Collider in the period $2002-2007$ were presented at the QCD 2008 conference \\ (Montpellier, France). These results include measurements of the mixing phase $\phi_{s}^{J / \psi \phi}$, decay width difference \\ $\Delta \Gamma_{s}$, and CP violation parameters in the $B_{s}$ and $B_{u}$ decays.
}

\section{Introduction}

The CDF and DØ experiments have successfully collected data since start of the Run II at the Tevatron Collider in 2001. The presented results correspond to an integrated luminosity of up to $2.8 \mathrm{fb}^{-1}$ at each experiment. The large $B$-meson production cross-section allows enough data to be collected to study the tiny effects of $\mathrm{CP}$-violation in $B$ systems. In particular, the results described in this paper cover measurements of the mixing phase $\phi_{s}^{J / \psi \phi}$, decay width difference $\Delta \Gamma_{s}$, semileptonic asymmetry $a_{s}^{s l}$ in the $B_{s}$ system and direct $\mathrm{CP}$ violation in the $B_{s}$ and $B_{u}$ systems.

A theoretical overview of $B_{s}-\bar{B}_{s}$ mixing is given in [12]. Flavour eigenstates of $B_{s}$ meson propagate according to the Schrödinger equation:

$i \frac{d}{d t}\left(\begin{array}{c}\left|B_{s}^{0}(t)\right\rangle \\ \left|\bar{B}_{s}^{0}(t)\right\rangle\end{array}\right)=\left(M_{s}-i \frac{\Gamma_{s}}{2}\right)\left(\begin{array}{l}\left|B_{s}^{0}(t)\right\rangle \\ \left|\bar{B}_{s}^{0}(t)\right\rangle\end{array}\right)$

The physical eigenstates $\left|B_{H}\right\rangle$ and $\left|B_{L}\right\rangle$ have different masses and lifetimes:

$\Delta M_{s}=M_{H}^{s}-M_{L}^{s}=2\left|M_{12}^{s}\right|$,

$\Delta \Gamma_{s}=\Gamma_{L}^{s}-\Gamma_{H}^{s}=2\left|\Gamma_{12}^{s}\right| \cos \phi_{s}$

where

$\phi_{s}=\arg \left(-M_{12}^{s} / \Gamma_{12}^{s}\right)$

is the CP phase. The CP phase for the $B_{s}$ system is very small in the Standard Model $(\mathrm{SM}): \phi_{s}^{S M} \approx$ 0.004. The review 2 predicts that the CP phase $\phi_{s}$ is the most sensitive to the new physics effects. The semileptonic asymmetry defined as

$a_{s l}^{s}=\frac{N\left(\bar{B}_{s} \rightarrow f\right)-N\left(B_{s} \rightarrow \bar{f}\right)}{N\left(\bar{B}_{s} \rightarrow f\right)+N\left(B_{s} \rightarrow \bar{f}\right)}$,

where $f$ corresponds to direct $B_{s}$ decays $B_{s} \rightarrow f$ (e.g. $\left.D_{s}^{-} l^{+} \nu_{l}\right)$, is related to the $\mathrm{CP}$ phase:

$a_{s l}^{s}=\frac{\Delta \Gamma_{s}}{\Delta M_{s}} \tan \phi_{s}$.

Measurements of all four parameters in the equation 6 allow testing of the SM predictions with high sensitivity. The mixing phase $\beta_{s}$ with the $\mathrm{SM}$ prediction $\beta_{s}^{S M}=\arg \left(-\left(V_{t s} V_{t b}^{*}\right) /\left(V_{c s} V_{c b}^{*}\right)\right) \approx$ 0.02 measured in decays $B_{s} \rightarrow J / \psi \phi$ is also related to the phase $\phi_{s}$ (see note in [3]). In paper [4] the notation $\phi_{s}=-2 \beta_{s}$ is used, but to distinguish this phase from the $\mathrm{CP}$ phase $\phi_{s}$ defined above in equation 4 the notation $\phi_{s}^{J / \psi \phi}=-2 \beta_{s}^{J / \psi \phi}$ as in [5] is preferable. The new physics contribution could be described by the phase $\phi_{s}^{N P}$ which modifies the original mixing phases in the following way: $\phi_{s}=\phi_{s}^{S M}+\phi_{s}^{N P}$ and $\beta_{s}=\beta_{s}^{S M}-\phi_{s}^{N P} / 2$.

2. $\phi_{s}^{J / \psi \phi}$ from $B_{s} \rightarrow J / \psi \phi$ 4, 6

The system $J / \psi+\phi$ from $B_{s}$ decay represents an admixture of $\mathrm{CP}$-even and $\mathrm{CP}$-odd states. Angular analysis could be used to separate the $\mathrm{CP}$ components (see figure 1) and measure the lifetime of each component and phase $\phi_{s}^{J / \psi \phi}[7]$. Additional information on the initial state flavor of the $B_{s}$ meson helps resolve the sign ambiguity on $\phi_{s}^{J / \psi \phi}$ for a given $\Delta \Gamma_{s}$ and improves the precision of results. The CDF collaboration used the data 
sample corresponding to $1.7 \mathrm{fb}^{-1}$ (this result was updated recently using $2.8 \mathrm{fb}^{-1}$ data sample [8]) and the $\mathrm{D} \varnothing$ data sample corresponds to $2.8 \mathrm{fb}^{-1}$. Constraints on the strong phases $\delta_{i}$ from the $B_{d}$ system allow the sign ambiguity on $\Delta \Gamma_{s}$ to be resolved (see recent theoretical work [9]). The $\mathrm{CDF}$ and $\mathrm{D} \varnothing$ results obtained with different constraints, and compared with the SM prediction are shown in figure 2. The HFAG combination [5] gives two sets of the numerical results obtained with free phases $\delta_{i}$ :

- $\Delta \Gamma_{s}=0.154_{-0.070}^{+0.054} \mathrm{ps}^{-1}$, $\phi_{s}^{J / \psi \phi}=-2 \beta_{s}^{J / \psi \phi}=-0.77_{-0.37}^{+0.29} \mathrm{rad}$;

- $\Delta \Gamma_{s}=-0.154_{-0.054}^{+0.070} \mathrm{ps}^{-1}$, $\phi_{s}^{J / \psi \phi}=-2 \beta_{s}^{J / \psi \phi}=2.36_{-0.29}^{+0.37} \mathrm{rad}$.

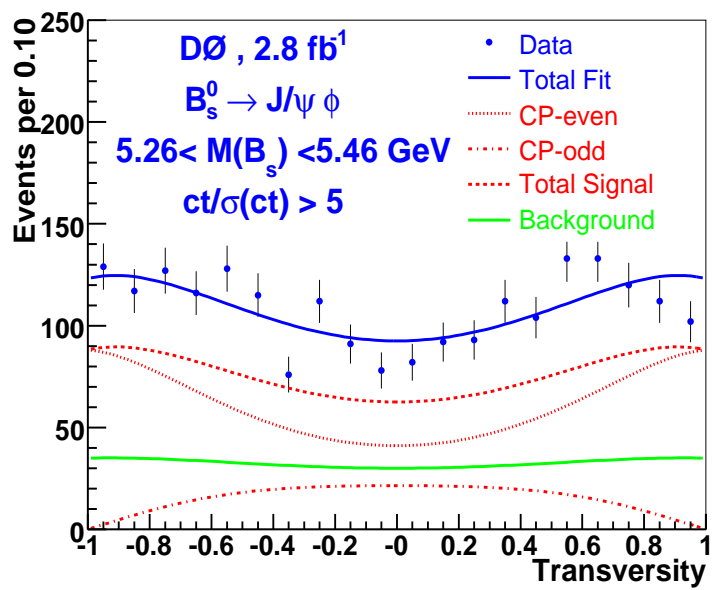

Figure 1. The transversity distribution for the signal-enhanced $J / \psi \phi$ subsample: non-prompt and signal mass region. The curves show: the signal contribution, dotted (red); the background, light solid (green); and total, dark solid (blue).

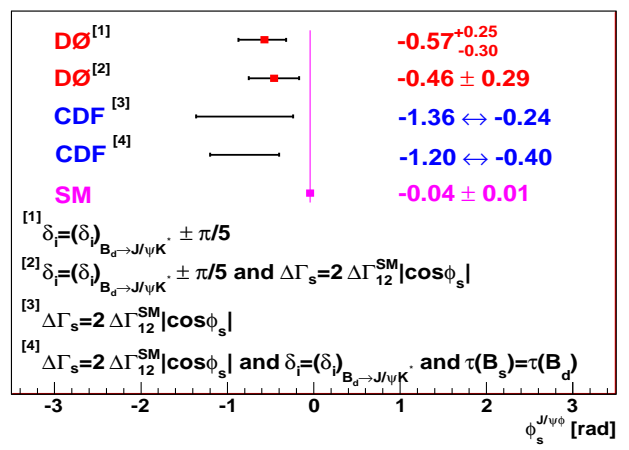

Figure 2. Comparison of the $\phi_{s}^{J / \psi \phi}$ results from $\mathrm{CDF}$ and $\mathrm{D} \varnothing$ with the SM prediction.

\section{3. $\Delta \Gamma_{s}$ from $B_{s} \rightarrow D_{s}^{(*)} D_{s}^{(*)}[10$}

The final state in $B_{s} \rightarrow D_{s}^{(*)} D_{s}^{(*)}$ decays is CPeven, although the theoretical uncertainty is not well understood (see for example the discussion in [2]). The branching ratio $\operatorname{Br}\left(B_{s} \rightarrow D_{s}^{(*)} D_{s}^{(*)}\right)$ could be used to calculate the decay width difference from the following equation derived in [1]:

$$
\begin{array}{r}
2 \cdot \operatorname{Br}\left(B_{s} \rightarrow D_{s}^{(*)} D_{s}^{(*)}\right) \approx \\
\Delta \Gamma_{s}^{C P}\left(\frac{1+\cos \phi_{s}}{2 \Gamma_{L}}+\frac{1-\cos \phi_{s}}{2 \Gamma_{H}}\right) .
\end{array}
$$

The efficient muon triggering system at $\mathrm{D} \varnothing$ provides access to these decays through the muons from semileptonic decays of $D_{s}$ mesons: $B_{s} \rightarrow$ $D_{s}^{(*)}(\rightarrow(\gamma) \phi \pi) D_{s}^{(*)}(\rightarrow(\gamma) \phi \mu \nu)$. Narrow invariant mass peaks from the $\phi \rightarrow K K$ decays help to ensure a clean data sample. The number of selected $D_{s} \phi \mu$ candidates was determined to be $N\left(D_{s} \phi \mu\right)=31.0 \pm 9.4$ and the resulting branching ratio: $\operatorname{Br}\left(B_{s} \rightarrow D_{s}^{(*)} D_{s}^{(*)}\right)=0.042 \pm$ 0.015 (stat.) \pm 0.017 (syst.). Assuming no new physics effects (the SM value of $\phi_{s}^{S M} \approx 0.004$ ) one can estimate the decay width difference from equation $7 \frac{\Delta \Gamma_{s}}{\Gamma_{s}}=0.088 \pm 0.030$ (stat. $) \pm$ 0.036(syst.). 


\section{CP violation}

\section{1. $B_{s}$ semileptonic decays [11, 12]}

The SM prediction for the semileptonic asymmetry in $B_{s}$ decays is very small [2]: $a_{s l}^{s}=$ $(2.06 \pm 0.57) \cdot 10^{-5}$. At the Tevatron the semileptonic asymmetry could be measured either from an inclusive di-muon sample, where

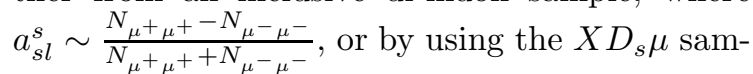
ple. The first method has very high statistical accuracy, but requires knowledge of asymmetries of other contributing processes in addition to the detector charge asymmetries. The second method has less statistical power but ensures that the major contribution to the asymmetry comes from the $B_{s}$ decays. The time-integrated untagged analysis at $\mathrm{D} \varnothing$ used the data sample $X D_{s}(\phi \pi) \mu$ corresponding to $1.3 \mathrm{fb}^{-1}$ to obtain the result $a_{s l}^{s}=0.0245 \pm 0.0193 \pm 0.0035$ [13] (this analysis was recently updated using a larger data sample, time dependence and initial-state tagging [14]). The description of the method used to determine the detector asymmetries at $\mathrm{D} \varnothing$ is given in 15 where the di-muon asymmetry result is presented. The combination of the two $\mathrm{D} \varnothing$ results is published in [12]: $a_{s l}^{s}=0.0001 \pm 0.0090$.

The preliminary CDF result obtained using the inclusive di-muon sample is shown in [11: $a_{s l}^{s}=$ $0.020 \pm 0.021 \pm 0.018$.

\section{2. $B_{s} \rightarrow K \pi[16]$}

The recent measurements of direct $\mathrm{CP}$ violation in the $B_{u}$ and $B_{d}$ systems led to the $K \pi$ puzzle (see for example [17]). It is therefore important to measure similar signatures in the $B_{s}$ system (see discussion in [18). The impact parameter triggers at CDF are used to collect the hadronic $B$-decays which are necessary for studies of $\mathrm{CP}$ eigenstates. Figure 3 shows the invariant mass distribution for 2 body hadronic decays at CDF assuming pion masses for the tracks. This sample allowed CDF to produce the only measurement of direct $\mathrm{CP}$ violation in the $B_{s}$ system: $A_{C P}^{B_{s} \rightarrow K^{-} \pi^{+}}=\frac{N_{\bar{B}_{s} \rightarrow K^{+} \pi^{-}}-N_{B_{s} \rightarrow K^{-} \pi^{+}}}{N_{\bar{B}_{s} \rightarrow K^{+} \pi^{-}}+N_{B_{s} \rightarrow K^{-} \pi^{+}}}=$ $0.39 \pm 0.15$ (stat.) \pm 0.08 (syst.). This result is in agreement with the theoretical predictions [19].

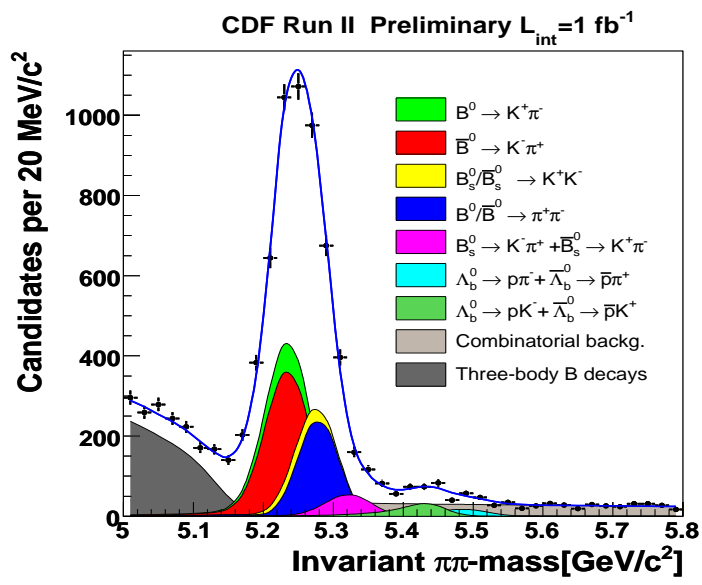

Figure 3. Invariant $\pi \pi$-mass of $B \rightarrow h^{+} h^{-}$candidates where $h$ is $K$ or $\pi$.

\section{3. $B^{ \pm} \rightarrow D^{0} K^{ \pm} 22$}

$\mathrm{CP}$ analysis of the decays $B^{ \pm} \rightarrow D^{0} K^{ \pm}$represents another example of using the impact parameter triggers at $\mathrm{CDF}$. The $\mathrm{CP}$ asymmetry is measured in these decays. This asymmetry is relevant for the determination of the CKM angle $\gamma$ (see [2324]). The $D^{0}$ meson decays either to CP eigenstates $K^{+} K^{-}\left(\pi^{+} \pi^{-}\right)$, or flavour eigenstate $K^{-} \pi^{+}$. The different modes were separated using invariant masses, momentum imbalance, total momentum and $d E / d x$ information. The measured asymmetries agree with the B-factory measurements 25[26] and have competitive precision: $A_{C P+}=\frac{B r\left(B^{-} \rightarrow D_{C P+}^{0} K^{-}\right)-B r\left(B^{+} \rightarrow D_{C P+}^{0} K^{+}\right)}{B r\left(B^{-} \rightarrow D_{C P+}^{0} K^{-}\right)+B r\left(B^{+} \rightarrow D_{C P+}^{0} K^{+}\right)}=$ $0.37 \pm 0.14$ (stat.) \pm 0.04 (syst.), $R=$ $\frac{B r\left(B^{-} \rightarrow D^{0} K^{-}\right)-B r\left(B^{+} \rightarrow \bar{D}^{0} K^{+}\right)}{B r\left(B^{-} \rightarrow D^{0} K^{-}\right)+B r\left(B^{+} \rightarrow D^{0} K^{+}\right)}=0.0745 \pm$ 0.0043 (stat.) \pm 0.0045 (syst.), $R_{C P+}=$ $\frac{\operatorname{Br}\left(B^{-} \rightarrow D_{C P+}^{0} K^{-}\right)+B r\left(B^{+} \rightarrow D_{C P+}^{0} K^{+}\right)}{\left(\operatorname{Br}\left(B^{-} \rightarrow D^{0} K^{-}\right)+\operatorname{Br}\left(B^{+} \rightarrow \bar{D}^{0} K^{+}\right)\right) / 2}=1.57 \pm$ 0.24 (stat.) \pm 0.12 (syst.).

\section{4. $B^{ \pm} \rightarrow J / \psi K^{ \pm}[20]$}

Highly efficient di-muon triggers allow for collection of large $J / \psi$ samples at Tevatron which are competitive with the B-factories in some of the $\mathrm{CP}$ violation measurements in $B_{u}$ and $B_{d}$ 
systems. The SM expectation of the direct $\mathrm{CP}$ asymmetry in $B^{ \pm} \rightarrow J / \psi K^{ \pm}$decays is small: $A_{S M}^{J / \psi K} \sim 0.003$ [21]. However, the new physics effects (for example a fourth generation) may enhance the asymmetry up to 0.01 . The $\mathrm{D} \varnothing$ experiment measured this asymmetry to be $A^{J / \psi K}=$ $+0.0075 \pm 0.0061$ (stat.) \pm 0.0027 (syst.).

\section{Conclusion}

So far, the Tevatron experiments CDF and DØ have provided the unique sources of the $\mathrm{CP}$ parameters in $B_{s}$ system. The complimentary measurements in $B_{u / d}$ systems are not only supporting the $B_{s}$ analyses but also providing results competitive with the B-factories. Interestingly though, the CP parameters in the $B_{s}$ system start showing some deviations from the SM predictions in addition to the $B_{u / d}$ puzzles. All analyses are statistically limited and the precision will improve in the near future.

The author thanks the organisers of the QCD08 Conference for a very interesting program and the physics analysis representatives from CDF and DØ for providing results.

\section{REFERENCES}

1. I. Dunietz, et al., Phys. Rev. D 63, 114015 (2001).

2. A. Lenz and U. Nierste, JHEP 0706:072 (2007).

3. A. Lenz, Nucl. Phys. Proc. Suppl. 177-178 : 81-86 (2008).

4. V. M. Abazov, et al., FERMILAB-PUB08/033-E; arXiv: 0802.2255 [hep-ex], submitted to PRL.

5. E. Barberio, et al., arXiv:0808.1297 [hep-ex].

6. T. Aaltonen, et al., Phys. Rev. Lett. 100, 121803 (2008).

7. A. S. Dighe, et al., Eur. Phys. J. C 6, 647 (1999).

8. The CDF Collaboration, CDF note 9458.

9. M. Gronau, J. L. Rosner, arXiv: 0808.3761 [hep-ph].

10. The DØ Collaboration, $D \varnothing$ note 5651.

11. The CDF Collaboration, CDF note 9015.
12. V. M. Abazov, et al., Phys. Rev. D 76, 057101 (2007).

13. V. M. Abazov, et al., Phys. Rev. Lett. 98, 151801 (2007).

14. The DØ Collaboration, DØ note 5730.

15. V. M. Abazov, et al., Phys. Rev. D 74, 092001 (2006).

16. The CDF Collaboration, CDF note 8579.

17. C.-W. Chiang, arXiv:0808.1336 [hep-ph].

18. R. Fleischer, Eur. Phys. J. C 52, 267 (2007).

19. C.-W. Chiang, et al., Phys. Lett. B664: 169173 (2008).

20. V. M. Abazov, et al., Phys. Rev. Lett. 100, $211802(2008)$.

21. W.-S. Hou, et al., arXiv: hep-ph/0605080.

22. The CDF Collaboration, CDF note 9109.

23. M. Gronau and D. London, Phys. Lett. B 253, 483 (1991).

24. M. Gronau and D. Wyler, Phys. Lett. B 265, 172 (1991).

25. B. Aubert, et al., Phys. Rev. D 77, 111102 (2008).

26. K. Abe, et al., Phys. Rev. D 73, 051106 (2006). 